\title{
NON-ALLELIC INTERACTIONS AND GENETIC ISOLATION IN WILD POPULATIONS OF ASPERGILLUS NIDULANS
}

\author{
A. C. BUTCHER * \\ Department of Genetics, The University, P.O. Box 363, Birmingham, 15
}

Received 1.i.69

\section{INTRODUCTION}

Heterokaryosis, the bringing together of unlike nuclei into the same cytoplasm, has been demonstrated in many fungi (see Davis, 1966; Caten and Jinks, 1966). In fungi imperfecti it is an essential step in the parasexual cycle, which is the sole means whereby genetic exchange can be achieved. In these fungi, therefore, heterokaryon incompatibility, as demonstrated in Aspergillus nidulans by Grindle $(1963 a, b)$ would seem to be a barrier to genetic exchange. Many sexual fungi are homothallic and it has been suggested that, in these, heterokaryon incompatibility would provide a barrier to genetic exchange via sexual and parasexual cycles alike (Grindle, $1963 b$; Jinks and Grindle, 1963; Caten and Jinks, 1966). Since heterokaryon incompatibility in Aspergillus nidulans, at least, is consistent with genotypic variation (Grindle, $1963 b$; Jinks, Caten, Simchen and Croft, 1966), it appears that within natural populations of this fungus there is, in all probability, a high degree of genetic isolation.

On the other hand, sexual outcrossing between wild isolates of $A$. nidulans which are heterokaryon incompatible has been reported initially by Jinks, Caten, Simchen and Croft (1966) and since by Butcher (1968), who has demonstrated that sexual outcrossing seems to be possible between all wild isolates to a greater or lesser extent, regardless of heterokaryon incompatibility. Jinks et al. (1966) found, however, evidence of reduced vigour in growth rate (presumably a fitness character) amongst the progeny of crosses between heterokaryon incompatible parents and expressed a doubt as to whether such progeny would be able to establish themselves in the natural environment in competition with their more vigorous parents.

It is the purpose of the work described in this paper to investigate more fully the comparative vigour of parents and progeny and to relate this to variation in genotype. Two approaches have been adopted. Firstly, a crossing experiment designed to provide estimates of additive and nonadditive variation in wild populations, and, secondly, data has been collected from a wide range of unrelated crosses and the relative performances of parents and their progenies compared.

\section{Materials AND methods}

(i) Crossing technique

The technique employed is that previously described by Butcher (1968). Matings were made by incubating mixed cultures of the two parents, obtained by spreading a mixed conidial suspension over the surface of a plate of minimal medium. Several perithecia were sampled from areas of the culture where conidial heads of both parental types were well intermingled,

* Present address: Sub-department of Genetics, The Queen's University of Belfast, David Keir Building, Belfast 9, N. Ireland.

2 R 2 
cleaned by rolling over an agar surface and squashed, thereby liberating a pool of ascospores. A sample of ascospores from each perithecium was spread over the surface of a clean plate and incubated. As all crosses were between one parent with green (wild type) conidia and one parent with yellow or white conidia, the progeny from a perithecium of hybrid origin could be instantly recognised. Samples of the individual colonies resulting from the germination of ascospores from such a perithecium were taken at random. In any one cross all the ten progeny sampled were taken from a single perithecium.

\section{(ii) Growth rate determinations}

The growth rate measurements were made using standard, $85 \mathrm{~mm}$. diameter, flat bottomed petri dishes containing $20 \mathrm{ml}$. of minimal agar. All incubations were carried out at $25^{\circ} \mathrm{C}$.

\section{TABLE 1}

Details of the wild isolates used in diallel crossing programme

\begin{tabular}{cclc} 
Isolate & h-c group & \multicolumn{1}{c}{ Origin } & Mutant used \\
55 & B & Farmyard compost heap, Dudley, Worcs. & $y$ \\
56 & A & Farmyard compost heap, Dudley, Worcs. & $w$ \\
60 & A & Woodland soil, Epping Forest, Essex & $y$ \\
61 & B & Woodland soil, Epping Forest, Essex & $y$
\end{tabular}

The character measured was linear growth rate across the surface of the plate as represented by the increase in diameter of a colony resulting from a point inoculation at the centre of the plate. Four days' incubation was allowed for germination to occur and for each colony to enter the linear phase of growth. Two extended diameters were then drawn in indian ink at right angles on the base of the plate. The position of the growing edge of the colony was marked along each diameter using a lamp, shining from behind the plate, to illuminate the growing edge. The plates were then reincubated for three days and the new positions of the growing edge marked along each diameter. The sum of the increased in the two diameters over the three day period was taken as a measure of growth rate for the purposes of analysis.

All plates were incubated in a randomised block design. The progeny and parents in any one experiment being represented by a single colony in each of two or three blocks.

\section{(iii) Choice of isolates and crossing programme}

Four wild isolates were used in the crossing experiment, one belonging to h-c group A and one to h-c group B from each of two different geographical locations (see table 1). In addition a U.V. induced derivative strain of each isolate having either yellow $(y)$ or white $(w)$ conidia was used in order to facilitate the recognition of hybrid perithecia. A $4 \times 4$ set of diallel crosses was produced by making all possible crosses between green (wild type) and yellow or white spored strains. The resulting 16 crosses can be grouped into 4 distinct types:

(a) Selfs, i.e. between a wild type isolate and its own mutant derivative, e.g. $55 \times 55 y$ (GL-type crosses). 
(b) Crosses between heterokaryon compatible isolates from different locations, e.g. $55 \times 61 y$ (G-type crosses).

(c) Crosses between heterokaryon incompatible isolates from the same location, e.g. $55 \times 56 w$ (L-type crosses).

(d) Crosses between heterokaryon incompatible isolates from different locations, e.g. $55 \times 60 y$ (O-type crosses).

Such a design provides information about the differences between h-c groups and between the sources of the isolates. If the analysis of variance derived by Hayman (Hayman, 1954) is applied it is possible to obtain estimates of both additive and non-additive variation between crosses. Two separate $2 \times 2$ diallel tables can be obtained by summing firstly over h-c groups and secondly over sources of isolates, which allow estimates of additive and non-additive variation between $h$-c groups, between sources and within h-c groups within sources.

\section{Results}

(i) Diallel cross

The results of the crosses are summarised in table 2 as the mean increase in diameter per day after summing over blocks. Also given in this table are the overall means of the four types of crosses. The means of both the L- and

TABLE 2

Mean progeny growth rate (mm./day) and classification of the individual crosses of the diallel

Spore-colour
mutants $\left\{\begin{array}{ccccc}55 y & 5 \cdot 900 & 5 \cdot 271 & 5 \cdot 225 & 6 \cdot 021 \\ & (\mathrm{GL}) & (\mathrm{L}) & (\mathrm{O}) & (\mathrm{G}) \\ & & & & \text { Wild types } \\ 56 w & 4 \cdot 846 & 4 \cdot 887 & 4 \cdot 854 & 5 \cdot 279 \\ & (\mathrm{~L}) & (\mathrm{GL}) & (\mathrm{G}) & (\mathrm{O}) \\ & & & & \\ 60 y & 4 \cdot 771 & 5 \cdot 112 & 4 \cdot 812 & 4 \cdot 604 \\ & (\mathrm{O}) & (\mathrm{G}) & (\mathrm{GL}) & (\mathrm{L}) \\ & & & & \\ 61 y & 5 \cdot 558 & 4 \cdot 833 & 4 \cdot 237 & 5 \cdot 717 \\ & (\mathrm{G}) & (\mathrm{O}) & (\mathrm{L}) & (\mathrm{GL})\end{array}\right.$

Overall means of the four types of cross (mm./day)

$\begin{array}{cccc}\text { GL } & \text { G } & \text { L } & \text { O } \\ 5 \cdot 329 & 5 \cdot 386 & 4 \cdot 740 & 5 \cdot 027\end{array}$

For full explanation of the classification symbols see text.

O-type crosses are lower than the mean of the GL-type which is in turn slightly lower than that of the G-type crosses. These results are in general accord with the findings of Jinks et al. (1966).

The analysis of variance adopted tests whether or not these differences are significant and have any genetic basis. It should be noted that $A$. nidulans is a haploid organism and that the diallel was constructed on the basis of the conidial colour of the parents and not on the basis of their sex. As a result the individual items in the analysis have different meanings from those of the 
conventional diallel analysis. Although items " $a$ " and " $b$ " still test additive and non-additive variation respectively, dominance effects are not included in the latter, as they cannot occur in the haploid system. Items " $c$ " and " $d$ " both test the effects of incorporating conidial colour markers into wild genotypes. They have been pooled to give a single " $c+d$ " item which tests reciprocal effects arising from the spore colour difference. It is therefore regarded as an additional error item. Table 3 is a summary of the significant items in the full analysis. All of the variation between crosses is attributable to differences between h-c groups and between sources, but not to reciprocal effects, and as such is due solely to genetically meaningful effects.

Additive variation results from heritable differences in the rate of growth between the parental isolates. Significant additive variation between h-c groups means that there are genetic differences between the isolates belonging to the different groups. Similarly, significant variation between sources means that there are genetic differences between the isolates collected from different locations.

It is non-additive variation, however, which is of most interest in the present study. As dominance is " ruled out" all of the non-additive variation must be due to interactions between non-allelic genes. Such interactions in the GL- and G-type crosses should show up as one of the within h-c group " $b$ " items. Neither is, in fact, significant. There is, therefore, no evidence of non-allelic interactions between heterokaryon compatible parents. There is, however, significant non-additive variation between h-c groups and thus non-allelic interactions occur in the L- and O-type crosses, i.e. in crosses between heterokaryon incompatible parents.

Table 3 contains estimates of the variance components for each of the significant items calculated by the formulae of Wearden (1964) for a model involving reciprocal effects and random sampling. These estimates clearly show that most of the variation encountered is due to differences between progeny within crosses. This is to be expected from the design of the experiment. Of the variation between crosses, most can be attributed to nonallelic interactions in crosses between h-c groups. Indeed, nearly five times as much variation is due to this cause as is due to additive differences in the growth rates of the two h-c groups. Genetic differences between isolates from different sources account for less than 2 per cent. of the total variation; less than that accounted for by the single significant block interaction. The significance of this latter item is probably fortuitous and it has no genetical importance.

It is clear, therefore, that non-allelic interactions are of marked importance in determining the growth rates of progeny from crosses between heterokaryon compatible individuals. That these interactions result in a net increase in growth rate has already been demonstrated by the overall means of the four types of crosses (table 2).

\section{(ii) Unrelated crosses}

To complement the data on the occurrence of non-allelic interactions in the diallel set of crosses, data was collected from a number of crosses between pairs of wild isolates which were not necessarily related in origin or h-c grouping. The criterion for the selection of these crosses was the availability of relevant information. The six crosses described by Jinks et al. (1966) are 


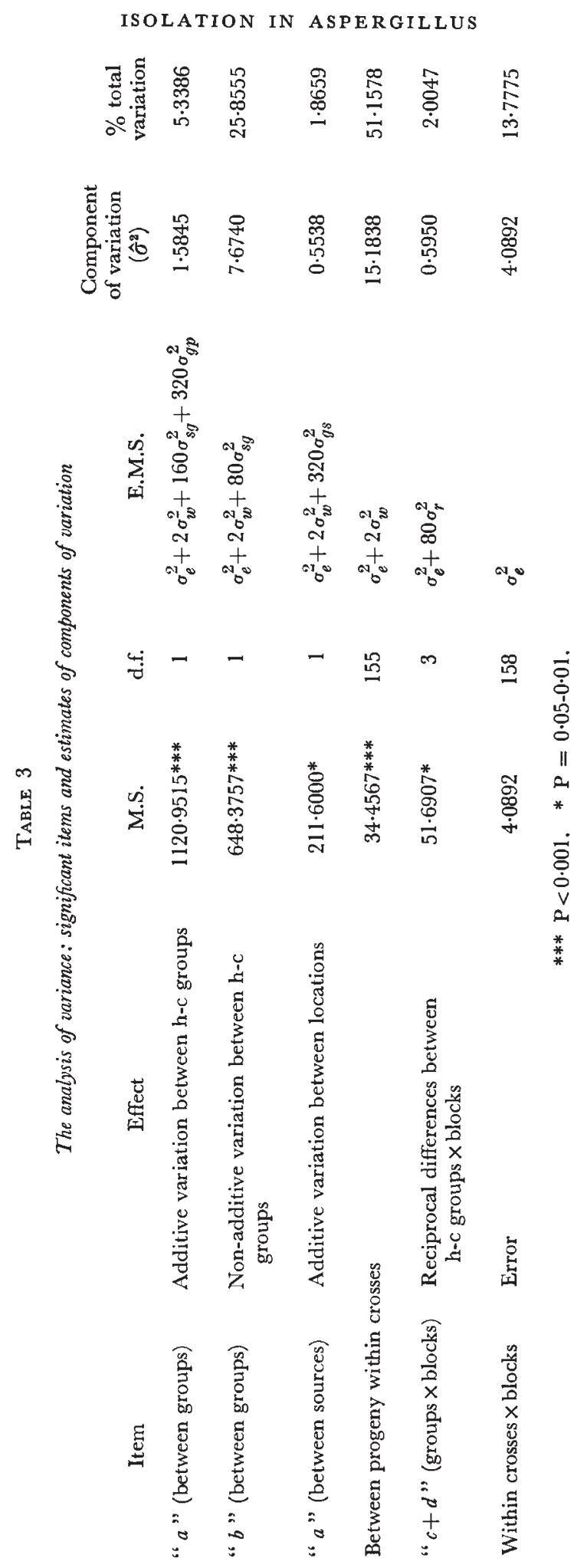


included and a wide range of heterokaryon compatibility relationships is represented.

The basic aim in considering these data is to investigate any possible relationships between genetic differences between parents and the occurrence of non-allelic interactions. The manifestation of such non-allelic interactions is measured by the difference between the mean growth rate of the parents and the mean growth rate of their progeny ([i], see section (iii) below). Genetic differences between the parents are expressed in the degree of genetic variation amongst the progeny $\left(s_{g}^{2}\right)$.

Mean growth rates of parents and progeny, and estimates of $s_{g}^{2}$ and [i], for each of the crosses, are given in table 4 .

TAble 4

Details of the seventeen unrelated crosses

\begin{tabular}{|c|c|c|c|c|}
\hline Cross & $\begin{array}{l}\text { Mean growth } \\
\text { rate of parents } \\
\text { (mm./day) }\end{array}$ & $\begin{array}{l}\text { Mean growth } \\
\text { rate of progeny } \\
\text { (mm./day) }\end{array}$ & $\begin{array}{c}\text { Genetic component of } \\
\text { between progeny } \\
\text { variance } \\
\left(s_{g}^{2}\right)\end{array}$ & {$[i]$} \\
\hline $43 \times 43 y$ & $6 \cdot 00$ & $5 \cdot 93$ & 0.015 & +0.07 \\
\hline $40 \times 43 y$ & $6 \cdot 60$ & $6 \cdot 76$ & $0 \cdot 023$ & -0.16 \\
\hline $139 \times 43 y$ & $6 \cdot 00$ & $6 \cdot 57$ & $0 \cdot 098$ & -0.57 \\
\hline $7 y, \mathrm{pro}_{4} \times 43 \mathrm{an}$ & $5 \cdot 30$ & $4 \cdot 30$ & $0 \cdot 327$ & $+1 \cdot 00$ \\
\hline $9 y, b i \times 43 a n$ & $6 \cdot 30$ & $5 \cdot 28$ & $0 \cdot 267$ & +1.02 \\
\hline $114 \times 37 y$ & $4 \cdot 0$ & $4 \cdot 04$ & 0.283 & +0.46 \\
\hline $99 \times 106 y$ & $5 \cdot 14$ & $5 \cdot 26$ & $0 \cdot 126$ & -0.12 \\
\hline $106 \times 99 y$ & $5 \cdot 54$ & $5 \cdot 45$ & $0 \cdot 299$ & +0.09 \\
\hline $119 \times 106 y$ & $5 \cdot 02$ & $5 \cdot 00$ & 0.174 & +0.02 \\
\hline $106 \times 119 y$ & $5 \cdot 65$ & $5 \cdot 39$ & $0 \cdot 206$ & +0.26 \\
\hline $26 \times 1 y$ & $5 \cdot 66$ & $5 \cdot 71$ & $0 \cdot 013$ & -0.05 \\
\hline $99 \times 31 y$ & 4.55 & $4 \cdot 48$ & $0 \cdot 139$ & +0.07 \\
\hline $31 \times 31 y$ & 4.53 & $4 \cdot 45$ & $0 \cdot 029$ & +0.08 \\
\hline $99 \times 99 y$ & 4.96 & $4 \cdot 89$ & 0.091 & +0.07 \\
\hline $99 \times 119 y$ & 4.98 & $5 \cdot 21$ & $0 \cdot 174$ & -0.23 \\
\hline $99 \times 182 y$ & $4 \cdot 71$ & $4 \cdot 81$ & $0 \cdot 120$ & $-0 \cdot 10$ \\
\hline $1 \times 31 y$ & $5 \cdot 20$ & $4 \cdot 71$ & $0 \cdot 178$ & +0.49 \\
\hline
\end{tabular}

(iii) Interpretation of the relationship between parents and progeny

The expected relationships between parental and progeny means and the variation amongst the progeny can be expressed in terms of a biometrical model. Thus Jinks has shown (personal communication) that the expected phenotypes may be written as

and

$$
\begin{aligned}
& \bar{P}_{1}=m+[d]+[i] \\
& \bar{P}_{2}=m-[d]+[i]
\end{aligned}
$$

where $P_{1}$ is the parent with the larger mean,

$P_{2}$ is the parent with the smaller mean,

$m$ is the origin from which the gene effects are measured,

$[d]$ is the contribution of additive gene effects to the mean phenotype,

$[i]$ is the contribution of non-additive gene effects to the mean phenotype.

(See Jinks and Jones, 1958.) 
The mid-parental value is then

$$
\frac{1}{2}\left(\bar{P}_{1}+\bar{P}_{2}\right)=m+[i]
$$

and $[d]$ is estimated as $\frac{1}{2}\left(\bar{P}_{1}-\bar{P}_{2}\right)$.

The mean phenotype of the progeny derived from a cross between $P_{1}$ and $P_{2}$ is then

$$
\bar{F}_{1}=m \text {. }
$$

Hence the difference between the mid-parental value and the progeny mean is

$$
\frac{1}{2}\left(\bar{P}_{1}+\bar{P}_{2}\right)-F_{1}=[i] .
$$

[i] is zero in the absence of non-allelic interactions, positive if $\frac{1}{2}\left(\ddot{P}_{1}+\tilde{P}_{2}\right)>\bar{F}_{1}$ and negative if $\frac{1}{2}\left(\bar{P}_{1}+\bar{P}_{2}\right)<F_{1}$. Thus reduced vigour amongst progeny in comparison to their parents indicates the presence of positive non-allelic interactions and increased vigour amongst progeny indicates negative interactions.

The expected contribution of the additive and interaction gene effects to the variance among the $F_{1}$ progeny is similarly

$$
D+I
$$

where $D=\Sigma\left(d^{2}\right)$,

$$
I=\Sigma\left(i^{2}\right) \text {. }
$$

In the absence of interactions this reduces to $D$.

Comparison of $s_{g}^{2}$ and [i] reveals a significant positive correlation $\left(r_{(16)}=0.6472, \mathrm{P}=0.01-0.001\right)$. Thus the vigour of progeny relative to that of their parents decreases as genetic differences between the parents increase. The data given in table 4 and the graph of [i] against $s_{g}^{2}$ (fig. 1) show that several crosses gave negative values of [i]. It is clear, however, that when $s_{g}^{2}$ has a value of zero [i] too must theoretically be zero, but not vice versa. This is because when $s_{g}^{2}$ is zero both parents and their progeny will have identical genotypes and no interactions are possible. A lack of interactions is not, however, associated with parents and progeny being identical. It could well be therefore, that a curvilinear relationship exists between [i] and $s_{g}^{2}$. The quadratic regression of [i] against $s_{g}^{2}$ (table 5) tests such a relationship. It can be seen from table 5 that a significant regression does exist which gives the sample regression line drawn in fig. 1 .

Certain conclusions can be drawn from this regression. First when $s_{g}^{2}=0$, the value of $[i]$ predicted by the regression is -0.0210 , which does not differ significantly from zero $\left(t_{(14)}=0.0599, \mathrm{P}>0.9\right)$. There is no evidence, therefore, that the regression line does not pass through the origin.

Second, the regression can be used to test whether or not the apparent presence of negative non-allelic interactions in some crosses has any real significance. This can be achieved by testing whether or not the value of [i] at the upper limit of the 5 per cent. confidence belt is ever negative. If it is assumed that the minimum predicted values of [i] on the sample regression line and upper 5 per cent. confidence limit occur at the same value of $s_{g}^{2}$, the appropriate test is of the significance of the deviation of the minimum predicted value of $[i]$ on the sample regression line from zero. The regression 
predicts a minimum value of $[i]$ of 0.0911 which does not differ significantly from zero $\left(t_{(14)}=0.2874, \mathrm{P}=0.8-0.7\right)$. Hence this particular regression provides no evidence of any significant negative interactions.

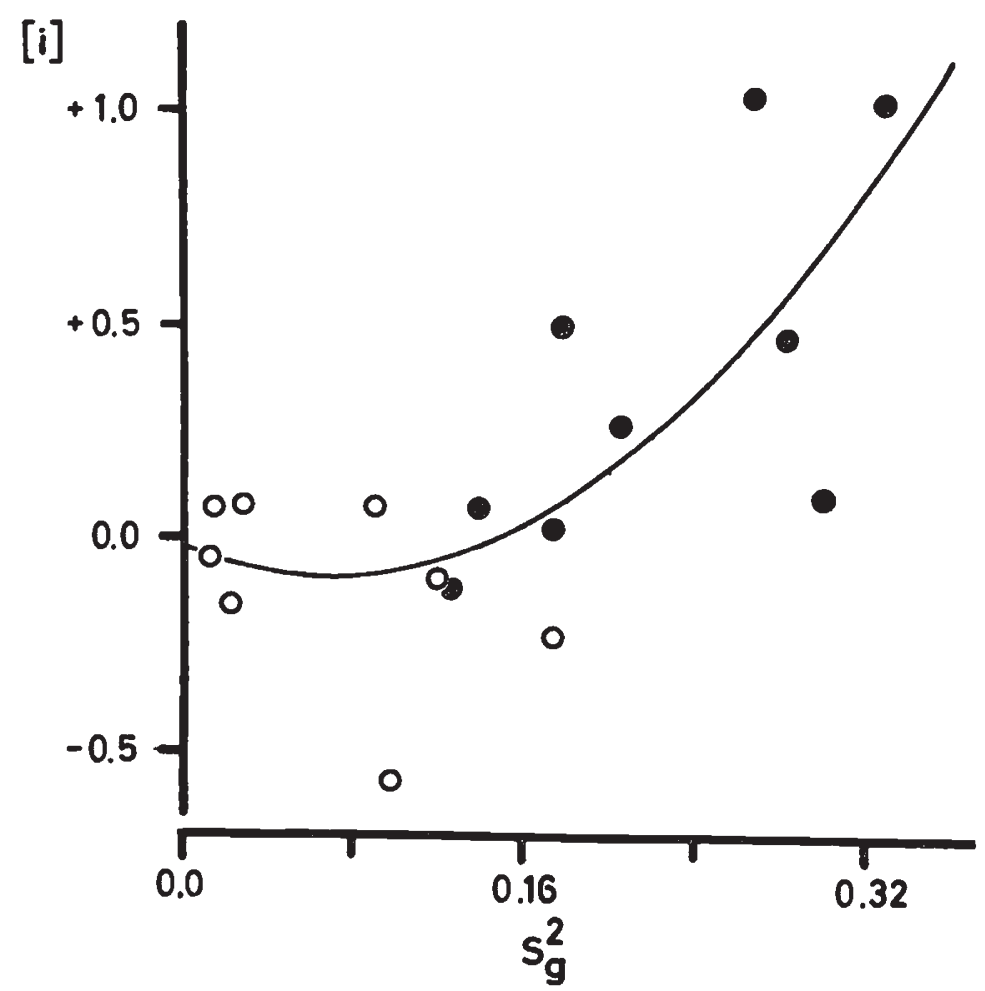

FIG. 1.-The contribution of non-allelic interaction to the parental phenotypes ([i]) plotted against the genetic component of the between progeny variance $\left(s_{g}^{2}\right)$ for seventeen unrelated crosses between wild isolates of $A$. nidulans. Open circles represent crosses between heterokaryon compatible isolates and closed circles represent crosses between heterokaryon incompatible isolates.

It would be entirely wrong to assume that a quadratic regression of [i] upon $s_{g}^{2}$ fully describes the relationship between [i] and $s_{g}^{2}$. It does however agree with theoretical expectation in that it almost passes through the origin

Table 5

\begin{tabular}{|c|c|c|}
\hline Item & d.f. & M.S. \\
\hline $\begin{array}{l}\text { Regression } \\
\text { Residual }\end{array}$ & $\begin{array}{r}2 \\
14\end{array}$ & $\begin{array}{l}0.711953^{* *} \\
0.089777\end{array}$ \\
\hline
\end{tabular}

and does show that a non-linear relationship can account for the results found. It is quite possible that the true relationship might well be a curve with [i] constant and zero when $s_{g}^{2}$ is small and increasing with $s_{g}^{2}$ when $s_{g}^{2}$ is large. 


\section{Discussion}

From results now at hand it seems clear that the breakdown of positive non-allelic interactions, resulting in reduced mean growth rate, is a common feature amongst sexual outcrosses between dissimilar wild isolates of $A$. nidulans and that the magnitude of this reduction is dependent upon the degree of difference between the genotypes of the parents. Thus the more dissimilar the parents, the greater is the extent of interaction breakdown and the less vigorous are the progeny. Although individual crosses were found where the progeny appeared to be more vigorous than their parents, particularly where the latter were heterokaryon compatible, no statistical evidence of significant negative non-allelic interactions could be found. On the other hand, the breakdown of positive non-allelic interactions was not particularly evident in crosses between relatively similar parents. It appears, from the results obtained, that a fair degree of divergence between parents is necessary before the absence of non-allelic interactions has any noticeable effect upon progeny vigour. If growth rate is assumed to be a fitness character, high growth rate can be considered to confer a selective advantage and thus the breakdown of non-allelic interactions causing a reduction in growth rate would presumably act as an isolating mechanism. It can be expected, therefore, that successful sexual outcrossing will only occur between genetically similar parents where such interactions will have very little effect.

In the absence of negative interactions a model of the type proposed by Simchen (1966) could explain the origin and nature of positive interactions. A situation is envisaged in which all individuals start from a common gene pool controlling growth rate. From this initial point, evolution occurs along several independent lines through the selection of mutations which increase growth rate by modifying the existing gene system. Thus the expression of each new mutation is dependent upon the genes already present. In this way many different polygenic systems can be built up. It is clear that if genetic reassortment occurs between any two of these systems, individual genes will be acting against genetic backgrounds different from those to which they are adapted, and will be unable to express themselves in their normal manner. The ultimate result of this will be a growth rate lower than the mean growth rate of the two parents. It also follows that the greater the divergence between the two parental systems the more drastic will be the effects of the absence of interactions on the progeny. This model clearly fits the experimental results obtained.

The model also supposes independent, divergent evolution which in turn requires some form of isolation. One of the suppositions of the model is that, initially, all individuals had a common gene pool controlling growth rate and were, therefore, not genetically isolated. The isolation therefore must, at least initially, have been physical in nature. Physical isolation can take many forms but in the present case one type, i.e. geographical isolation, can be ruled out since Grindle (personal communication) has shown an apparently sympatric distribution of h-c groups throughout England and Wales.

When considering soil-borne micro-organisms, however, gross geographical differences have virtually no significance in comparison with the vast environmental variation found over very small distances within the soil. Unfortunately the records pertaining to the origins of the wild isolates of 
$A$. nidulans are not detailed enough for conclusions to be drawn on distributions in relation to micro-environment and it may well be that different genotypes are isolated by adaption to different environmental conditions.

Extrapolation from experimental results obtained under laboratory conditions to any hypothesis about relationships in the natural environment is always speculative. Such extrapolation is particularly hazardous in the present case since it is not known whether sexual crossing and heterokaryosis do or do not occur in the soil. Indeed the stage of the life cycle in which the fungus usually exists in the soil is not as yet known. For this reason it is hard to argue cause and effect relationships with any degree of certainty.

Two types of barrier to free genetic exchange have now been demonstrated to occur amongst wild isolates of $A$. nidulans under laboratory conditions. The first prevents successful heterokaryosis, and thus parasexual recombination, and the second prevents successful sexual outcrossing. These two systems have several characteristics in common. First, both prevent genetic exchange between genetically dissimilar individuals while allowing exchange between genetically similar individuals, and therefore both favour inbreeding. Second, both types of barrier allow the initial exchange process to occur, i.e. hyphal anastomosis does appear to take place between heterokaryon incompatible isolates and sexual outcrossing resulting in viable progeny can occur between dissimilar individuals. Third, the barrier to sexual recombination acts through a selective disadvantage of the recombinant progeny, and it is possible that the barrier to heterokaryosis is also due to a selective disadvantage of, in this case, the heterokaryotic association. If this is so, then there is no evidence that the two barriers are not caused by the same interactions.

A. nidulans is not unique in having parallel systems restricting genetic exchange. Esser (1965) has shown, for example, that in another basically inbreeding ascomycete, Podospora anserina, there is a heterogenic sexual incompatibility system which also causes vegetative incompatibility. In this fungus the same four pairs of allelomorphs determine both heterokaryosis and sexual mating ability.

In situations such as these, it is reasonable to assume that inbreeding has distinct advangages over outbreeding and that the barriers to genetic exchange probably serve to maintain adaptive genotypes. With this assumption in mind, it might well be concluded that recent work (Butcher, 1968), showing that the rate of sexual outcrossing amongst wild isolates of $A$. nidulans varies according to the genotypes involved, indicates a possible evolutionary trend towards loss of sexual power. Especially as many Aspergilli closely related to $A$. nidulans have no functional sexual stage.

\section{Summary}

1. A $4 \times 4$ diallel crossing programme involving four wild isolates of A. nidulans from two h-c groups and from two remote soil samples was carried out. Growth rate determinations were made on the parents and progeny of the 16 crosses.

2. Non-additive variation due to the loss of positive non-allelic interactions was found in crosses between heterokaryon incompatible isolates, but only additive variation in crosses between heterokaryon compatible isolates.

3. Growth rate data from seventeen unrelated crosses between wild 
isolates are considered, and a positive correlation between non-allelic interaction and genetic differences between parents demonstrated.

4. A model for the origin and nature of the non-allelic interactions is discussed, together with the probable effects of these interactions on genetic exchange.

5. Comparisons are drawn between the barriers to genetic exchange caused by heterokaryon incompatibility and non-allelic interactions in sexual outcrosses.

Acknowledgments. - I am indebted to Professor J. L. Jinks for his advice and encouragement throughout the course of this work and for allowing me to quote his biometrical model. I wish to thank Dr J. H. Croft and Dr C. E. Caten for their constructive criticisms of this paper.

\section{References}

BUTCHER, A. c. 1968. The relationship between sexual outcrossing and heterokaryon incompatibility in Aspergillus nidulans. Heredity, 23, 443-451.

CATEN, C. E., AND JINKS, J. L. 1966. Heterokaryosis: its significance in wild homothallic Ascomycetes and fungi imperfecti. Trans. Br. Mycol. Soc., 49, 81-93.

DAvis, R. H. 1966. Heterokaryosis. In: The Fungi: An Advanced Treatise, Ainsworth, G. C. and Sussman, A. S. edits., Vol. II, 567-588. Academic Press, New York and London. ESSER, K. 1965. Heterogenic incompatibility. In: Incompatibility in Fungi, Esser, K. and Raper, J. R. edits., 6-13. Springer-Verlag, Berlin, Heidelberg, and New York.

GRINDLE, M. 1963a. Heterokaryon compatibility of unrelated strains in the Aspergillus nidulans group. Heredity, 18, 191-204.

GRINDLE, M. 1963b. Heterokaryon compatibility of closely related wild isolates of Aspergillus nidulans. Heredity, 18, 397-405.

HAYMAN, B. I. 1954. The analysis of variance of diallel crosses. Biometrics, 10, 789-809.

JINKS, J. L., CATEN, C. E., SIMCHEN, G., AND CROFT, J. H. 1966. Heterokaryon incompatibility and variation in wild populations of Aspergillus nidulans. Heredity, 21, 227-239.

JINKS, J. L., AND GRINDLE, M. 1963. The genetical basis of heterokaryon incompatibility in Aspergillus nidulans. Heredity, 18, 227-239.

JiNks, J. L., AND JONES, R. M. 1958. Estimation of the components of heterosis. Genetics, 43 , $223-234$.

PONTECORVO, G., ROPER, J. A., hemmons, L. M., MACDONALD, K. D., AND BUfton, A. J. W. 1953. The genetics of Aspergillus nidulans. Ad. Genet., 6, 141-238.

simchen, G. 1966. Monokaryotic variation and haploid selection in Schizophyllum commune. Heredity, 21, 241-263.

WEARDEN, s. 1964. Alternative analyses of the diallel cross. Heredity, 19, 669-680. 